(c) 2010 IEEE. Personal use of this material is permitted. Permission from IEEE must be obtained for all other uses, in any current or future media, including reprinting/republishing this material for advertising or promotional purposes, creating new collective works, for resale or redistribution to servers or lists, or reuse of any copyrighted component of this work in other works. 


\section{Optimal Joint Source and Relay Beamforming for Parallel MIMO Relay Networks}

\author{
Apriana Toding \\ Dept. Electrical Computer Engr. \\ Curtin University of Technology \\ Bentley, WA 6102, Australia \\ Muhammad R. A. Khandaker \\ Dept. Electrical Computer Engr. \\ Curtin University of Technology \\ Bentley, WA 6102, Australia
}

\author{
Yue Rong \\ Dept. Electrical Computer Engr. \\ Curtin University of Technology \\ Bentley, WA 6102, Australia \\ Email: y.rong@curtin.edu.au
}

\begin{abstract}
In this paper, we study the optimal structure of the source precoding matrix and the relay amplifying matrices for multiple-input multiple-output (MIMO) relay communication systems with parallel relay nodes. We show that both the optimal source precoding matrix and the optimal relay amplifying matrices have a beamforming structure. Using the optimal structure, a joint source and relay power loading algorithm is developed to minimize the mean-squared error (MSE) of the signal waveform estimation. Compared with existing algorithms for parallel MIMO relay networks, the proposed joint source and relay beamforming algorithm has a significant improvement in the system bit-error-rate performance.
\end{abstract}

\section{INTRODUCTION}

In order to establish a reliable wireless communication link, one needs to compensate for the effects of signal fading and shadowing. An efficient way to address this issue is to transmit signals through one or multiple relays [1]-[3]. When nodes in the relay system are installed with multiple antennas, we call such system multiple-input multiple-output (MIMO) relay communication system. Recently, MIMO relay communication systems have attracted much research interest and provided significant improvement in terms of both spectral efficiency and link reliability [4]-[15]. Many works have studied the optimal relay amplifying matrix for the sourcerelay-destination channel. In [7] and [8], the optimal relay amplifying matrix maximizing the mutual information (MI) between the source and destination was derived assuming that the source covariance matrix is an identity matrix. In [9] and [10], the relay amplifying matrix was designed to minimize the mean-squared error (MSE) of the signal waveform estimation at the destination.

A few research has studied the jointly optimal structure of the source precoding matrix and the relay amplifying matrix for the source-relay-destination channel. In [11], both the source covariance matrix and the relay amplifying matrix were jointly designed to maximize the source-destination MI. In [12] and [13], a unified framework was developed to jointly optimize the source precoding matrix and the relay amplifying matrix for a broad class of objective functions.

In [7]-[9] and [13]-[15], the optimal relay amplifying matrix was derived for two-hop MIMO relay networks with a single relay node. In [10] the authors investigated the optimal relay amplifying matrices for two-hop MIMO relay networks with multiple parallel relay nodes. However, the source precoding matrix was not optimized in [10]. In this paper, we propose a jointly optimal source and relay beamforming algorithm which minimizes the MSE of the signal waveform estimation for twohop MIMO relay communication systems with parallel relay nodes. Our results show that the optimal joint source and relay algorithm has a significant performance improvement over the relay only optimal algorithm developed in [10].

The rest of this paper is organized as follows. The system model is described in Section II. In Section III we study the optimal structure of the source and relay matrices. Section IV shows the simulation results. Conclusion are drawn in Section $\mathrm{V}$.

\section{SYSTEM MODEL}

Fig. 1 illustrates a two-hop MIMO relay communication system consisting of one source node, $K$ parallel relay nodes, and one destination node. We assume that the source and destination nodes have $N_{s}$ and $N_{d}$ antennas, respectively, and each relay node has $N_{r}$ antennas. The generalization to the system with different number of antennas at each relay node is straightforward. To efficiently exploit the system hardware, each relay node uses the same antennas to transmit and receive signals. Due to its merit of simplicity, we consider the amplifyand-forward scheme at each relay.

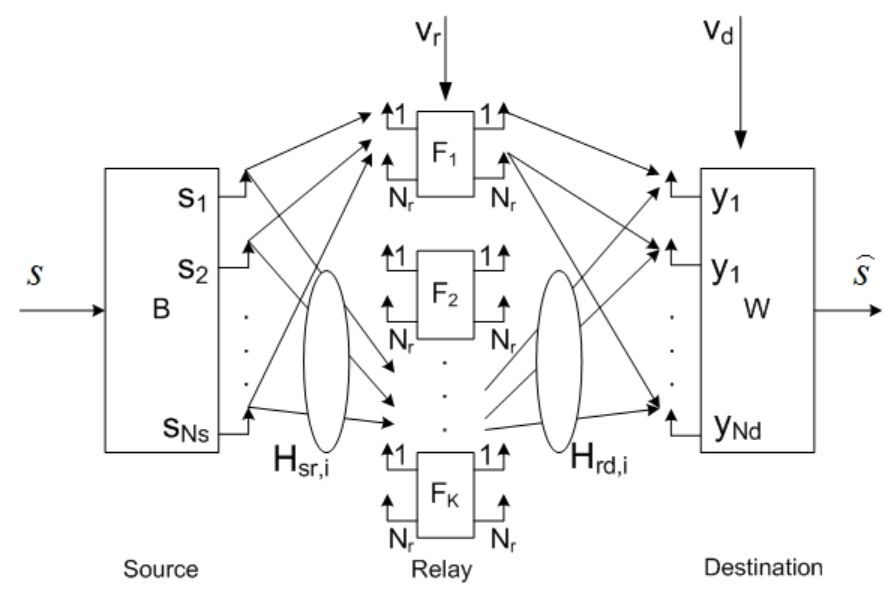

Fig. 1. Block diagram of a parallel MIMO relay communication system. 
The communication process between the source and destination nodes is completed in two time slots. In the first time slot, the $N_{b} \times 1$ modulated symbol vector $\mathbf{s}$ is linearly precoded as

$$
\mathbf{x}=\mathbf{B s}
$$

where $\mathrm{B}$ is an $N_{s} \times N_{b}$ source precoding matrix. Without any loss of transmitting power, we assume that $N_{b} \leq$ $\min \left(N_{s}, K N_{r}, N_{d}\right)$. The precoded vector $\mathbf{x}$ is transmitted to the relay nodes. The received signal at the $i$ th relay node can be written as

$$
\mathbf{y}_{r, i}=\mathbf{H}_{s r, i} \mathbf{x}+\mathbf{v}_{r, i}, \quad i=1, \cdots, K
$$

where $\mathbf{H}_{s r, i}$ is the $N_{r} \times N_{s}$ MIMO channel matrix between the source and the $i$ th relay node, $\mathbf{y}_{r, i}$ and $\mathbf{v}_{r, i}$ are the received signal and the additive Gaussian noise vectors at the $i$ th relay node, respectively.

In the second time slot, the source node is silent, while each relay node transmits the amplified signal vector to the destination node as

$$
\mathbf{x}_{r, i}=\mathbf{F}_{i, i} \mathbf{y}_{r, i} \quad i=1, \cdots, K
$$

where $\mathbf{F}_{i}$ is the $N_{r} \times N_{r}$ amplifying matrix at the $i$ th relay node. Thus the received signal vector at the destination node can be written as

$$
\mathbf{y}_{d}=\sum_{i=1}^{K} \mathbf{H}_{r d, i} \mathbf{x}_{r, i}+\mathbf{v}_{d}
$$

where $\mathbf{H}_{r d, i}$ is the $N_{d} \times N_{r}$ MIMO channel matrix between the $i$ th relay and the destination node, $\mathbf{y}_{d}$ and $\mathbf{v}_{d}$ are the received signal and the additive Gaussian noise vectors at the destination node, respectively.

Substituting (1)-(3) into (4), we have

$$
\begin{aligned}
\mathbf{y}_{d} & =\sum_{i=1}^{K} \mathbf{H}_{r d, i} \mathbf{F}_{i} \mathbf{H}_{s r, i} \mathbf{B s}+\mathbf{H}_{r d, i} \mathbf{F}_{i} \mathbf{v}_{r, i}+\mathbf{v}_{d} \\
& =\mathbf{H}_{r d} \mathbf{F} \mathbf{H}_{s r} \mathbf{B s}+\mathbf{H}_{r d} \mathbf{F} \mathbf{v}_{r}+\mathbf{v}_{d}
\end{aligned}
$$

where we define

$$
\begin{aligned}
\mathbf{H}_{s r} & \triangleq\left[\left(\mathbf{H}_{s r, 1}\right)^{T},\left(\mathbf{H}_{s r, 2}\right)^{T}, \cdots,\left(\mathbf{H}_{s r, K}\right)^{T}\right]^{T} \\
\mathbf{H}_{r d} & \triangleq\left[\mathbf{H}_{r d, 1}, \mathbf{H}_{r d, 2}, \cdots, \mathbf{H}_{r d, K}\right] \\
\mathbf{F} & \triangleq \operatorname{bd}\left[\mathbf{F}_{1}, \mathbf{F}_{2}, \cdots, \mathbf{F}_{K}\right] \\
\mathbf{v}_{r} & \triangleq\left[\left(\mathbf{v}_{r, 1}\right)^{T},\left(\mathbf{v}_{r, 2}\right)^{T}, \cdots,\left(\mathbf{v}_{r, K}\right)^{T}\right]^{T} .
\end{aligned}
$$

Here $(\cdot)^{T}$ denotes the matrix (vector) transpose, bd $(\cdot)$ stands for a block-diagonal matrix, $\mathbf{H}_{s r}$ is a $K N_{r} \times N_{s}$ channel matrix between the source node and all relay nodes, $\mathbf{H}_{r d}$ is an $N_{d} \times K N_{r}$ channel matrix between all relay nodes and the destination node, $\mathbf{v}_{r}$ is obtained by stacking the noise vectors at all the relays and $\mathbf{F}$ is the $K N_{r} \times K N_{r}$ block diagonal equivalent relay matrix. The diagram of the equivalent MIMO relay system described by (5) is shown in Fig. 2 .

By introducing

$$
\overline{\mathbf{F}} \triangleq \mathbf{H}_{r d} \mathbf{F}
$$

the received signal vector at the destination can be equivalently written as

$$
\mathbf{y}_{d}=\overline{\mathbf{F}} \mathbf{H}_{s r} \mathbf{B} \mathbf{s}+\overline{\mathbf{F}} \mathbf{v}_{r}+\mathbf{v}_{d}=\overline{\mathbf{H}} \mathbf{s}+\overline{\mathbf{v}}
$$

where we define $\overline{\mathbf{H}} \triangleq \overline{\mathbf{F}} \mathbf{H}_{s r} \mathbf{B}$ as the effective MIMO channel matrix of the source-relay-destination link, and $\overline{\mathbf{v}}$ as the equivalent noise with $\overline{\mathbf{v}} \triangleq \overline{\mathbf{F}} \mathbf{v}_{r}+\mathbf{v}_{d}$

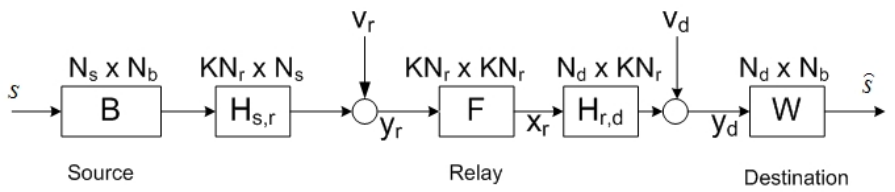

Fig. 2. Block diagram of the equivalent MIMO relay system.

\section{Minimal MSE Relay Design}

Using a linear receiver, the estimated signal waveform vector at the destination node is given by

$$
\hat{\mathbf{s}}=\mathbf{W}^{H} \mathbf{y}_{d}
$$

where $\mathbf{W}$ is an $N_{d} \times N_{b}$ weight matrix, and $(\cdot)^{H}$ denotes the matrix (vector) Hermitian transpose. The minimal MSE (MMSE) approach tries to find a weight matrix $\mathbf{W}$ that minimizes the statistical expectation of the signal waveform estimation given by

$$
\mathrm{MSE}=\operatorname{tr}\left(\mathrm{E}\left[(\hat{\mathbf{s}}-\mathbf{s})(\hat{\mathbf{s}}-\mathbf{s})^{H}\right]\right)
$$

where $\operatorname{tr}(\cdot)$ stands for the matrix trace, and $\mathrm{E}[\cdot]$ denotes statistical expectation. We assume that the source signal vector satisfies $\mathrm{E}\left[\mathbf{s s}^{H}\right]=\mathbf{I}_{N_{b}}$, where $\mathbf{I}_{n}$ is an $n \times n$ identity matrix, and all noises are independent and identically distributed with zero mean and unit variance. Substituting (7) into (8), we find that the $\mathbf{W}$ which minimizes (8) can be written as

$$
\mathbf{W}=\left(\overline{\mathbf{H}} \overline{\mathbf{H}}^{H}+\overline{\mathbf{C}}\right)^{-1} \overline{\mathbf{H}}
$$

where $(\cdot)^{-1}$ denotes the matrix inversion, and $\overline{\mathbf{C}}$ is the equivalent noise covariance matrix written as

$$
\overline{\mathbf{C}} \triangleq \overline{\mathbf{F}} \overline{\mathbf{F}}^{H}+\mathbf{I}_{N_{d}} \text {. }
$$

Using (9), it can be seen that the MSE is a function of $\overline{\mathbf{F}}$ and $\mathbf{B}$ and can be written as

$$
\mathrm{MSE}=\operatorname{tr}\left(\left[\mathbf{I}_{N_{b}}+\overline{\mathbf{H}}^{H} \overline{\mathbf{C}}^{-1} \overline{\mathbf{H}}\right]^{-1}\right) .
$$

The joint source and relay optimization problem is

$$
\begin{array}{ll}
\min _{\overline{\mathbf{F}}, \mathbf{B}} & \operatorname{tr}\left(\left[\mathbf{I}_{N_{b}}+\overline{\mathbf{H}}^{H} \overline{\mathbf{C}}^{-1} \overline{\mathbf{H}}\right]^{-1}\right) \\
\text { s.t. } & \operatorname{tr}\left(\mathbf{B B}^{H}\right) \leq P_{s} \\
& \operatorname{tr}\left(\overline{\mathbf{F}}\left[\mathbf{H}_{s r} \mathbf{B B}^{H} \mathbf{H}_{s r}^{H}+\mathbf{I}_{K N_{r}}\right] \overline{\mathbf{F}}^{H}\right) \leq P_{r}
\end{array}
$$

where (11) is the transmit power constraint at the source node, while (12) is the power constraint at the output of $\mathbf{H}_{r d}$ [10]. Here $P_{r}>0$ and $P_{s}>0$ are the corresponding power budget.

Let $\mathbf{H}_{s r}=\mathbf{U}_{s} \boldsymbol{\Lambda}_{s} \mathbf{V}_{s}^{H}$ denote the singular value decomposition (SVD) of $\mathbf{H}_{s r}$, where the dimensions of $\mathbf{U}_{s}, \boldsymbol{\Lambda}_{s}, \mathbf{V}_{s}$ 
are $K N_{r} \times K N_{r}, K N_{r} \times N_{s}, N_{s} \times N_{s}$, respectively. We assume that the main diagonal elements of $\boldsymbol{\Lambda}_{s}$ are arranged in a decreasing order. Using Theorem 1 in [13], the optimal structure of $\overline{\mathbf{F}}$ and $\mathbf{B}$ as the solution to the problem (10)-(12) is given by

$$
\overline{\mathbf{F}}=\mathbf{Q} \boldsymbol{\Lambda}_{f} \mathbf{U}_{s, 1}^{H}, \quad \mathbf{B}=\mathbf{V}_{s, 1} \boldsymbol{\Lambda}_{b}
$$

where $\mathbf{Q}$ is any $N_{d} \times N_{b}$ semi-unitary matrix with $\mathbf{Q}^{H} \mathbf{Q}=$ $\mathbf{I}_{N_{b}}, \mathbf{U}_{s, 1}$ and $\mathbf{V}_{s, 1}$ contain the leftmost $N_{b}$ columns of $\mathbf{U}_{s}$ and $\mathbf{V}_{s}$, respectively, $\boldsymbol{\Lambda}_{f}$ and $\boldsymbol{\Lambda}_{b}$ are $N_{b} \times N_{b}$ diagonal matrices. From (13) we see that the optimal $\overline{\mathbf{F}}$ and $\mathbf{B}$ have a beamforming structure. In fact, they jointly diagonalize the source-relay-destination channel $\overline{\mathbf{H}}$. Using (13), the joint source-relay optimization problem (10)-(12) becomes

$$
\begin{aligned}
\min _{\boldsymbol{\Lambda}_{f}, \boldsymbol{\Lambda}_{b}} & \operatorname{tr}\left(\left[\mathbf{I}_{N_{b}}+\left(\boldsymbol{\Lambda}_{f} \boldsymbol{\Lambda}_{s} \boldsymbol{\Lambda}_{b}\right)^{2}\left[\boldsymbol{\Lambda}_{f}^{2}+\mathbf{I}_{N_{b}}\right]^{-1}\right]^{-1}\right) \\
\text { s.t. } & \operatorname{tr}\left(\boldsymbol{\Lambda}_{b}^{2}\right) \leq P_{s} \\
& \operatorname{tr}\left(\boldsymbol{\Lambda}_{f}^{2}\left[\left(\boldsymbol{\Lambda}_{s} \boldsymbol{\Lambda}_{b}\right)^{2}+\mathbf{I}_{N_{b}}\right]\right) \leq P_{r} .
\end{aligned}
$$

Denoting $\lambda_{f, i}, \lambda_{s, i}, \lambda_{b, i}, i=1, \cdots, N_{b}$, as the main diagonal elements of $\boldsymbol{\Lambda}_{f}, \boldsymbol{\Lambda}_{s}, \boldsymbol{\Lambda}_{b}$, respectively, the optimization problem (14)-(16) can be equivalently written as

$$
\begin{aligned}
\min _{\left\{\lambda_{f, i}\right\},\left\{\lambda_{b, i}\right\}} & \sum_{i=1}^{N_{b}}\left(1+\frac{\left(\lambda_{f, i} \lambda_{s, i} \lambda_{b, i}\right)^{2}}{\lambda_{f, i}^{2}+1}\right)^{-1} \\
\text { s.t. } & \sum_{i=1}^{N_{b}} \lambda_{b, i}^{2} \leq P_{s} \\
& \sum_{i=1}^{N_{b}} \lambda_{f, i}^{2}\left[\left(\lambda_{s, i} \lambda_{b, i}\right)^{2}+1\right] \leq P_{r} \\
& \lambda_{b, i} \geq 0, \quad \lambda_{f, i} \geq 0, \quad i=1, \cdots, N_{b} .
\end{aligned}
$$

The problem (17)-(20) is nonconvex and a closed-form solution is intractable to obtain. In the following, we develop an iterative method to obtain a numerical solution of the optimal $\left\{\lambda_{f, i}\right\}$ and $\left\{\lambda_{b, i}\right\}$. Let us define

$$
\begin{aligned}
& a_{i} \triangleq \lambda_{s, i}^{2}, \quad x_{i} \triangleq \lambda_{b, i}^{2}, \\
& y_{i} \triangleq \lambda_{f, i}^{2}\left[\left(\lambda_{s, i} \lambda_{b, i}\right)^{2}+1\right], \quad i=1, \cdots, N_{b} .
\end{aligned}
$$

Then the optimization problem (17)-(20) can be equivalently rewritten as

$$
\begin{aligned}
\min _{\left\{x_{i}\right\},\left\{y_{i}\right\}} & \sum_{i=1}^{N_{b}}\left(1+\frac{\frac{a_{i} x_{i} y_{i}}{a_{i} x_{i}+1}}{1+\frac{y_{i}}{a_{i} x_{i}+1}}\right)^{-1} \\
\text { s.t. } & \sum_{i=1}^{N_{b}} x_{i} \leq P_{s} \\
& \sum_{i=1}^{N_{b}} y_{i} \leq P_{r} \\
& x_{i} \geq 0, \quad y_{i} \geq 0, \quad i=1, \cdots, N_{b} .
\end{aligned}
$$

For a fixed $\left\{y_{i}\right\}$ satisfying (24) and (25), the problem of optimizing $\left\{x_{i}\right\}$ can be written as

$$
\begin{array}{ll}
\min _{\left\{x_{i}\right\}} & \sum_{i=1}^{N_{b}} \frac{a_{i} x_{i}+y_{i}+1}{a_{i} x_{i} y_{i}+a_{i} x_{i}+y_{i}+1} \\
\text { s.t. } & \sum_{i=1}^{N_{b}} x_{i} \leq P_{s} \\
& x_{i} \geq 0, \quad i=1, \cdots, N_{b} .
\end{array}
$$

The Lagrangian function associated with the problem (26)-(28) can be written as

$$
\mathcal{L}=\sum_{i=1}^{N_{b}} \frac{a_{i} x_{i}+y_{i}+1}{a_{i} x_{i} y_{i}+a_{i} x_{i}+y_{i}+1}+\mu_{1}\left(\sum_{i=1}^{N_{b}} x_{i}-P_{s}\right)
$$

where $\mu_{1} \geq 0$ is the Lagrangian multiplier. Taking the derivative of (29) with respect to $x_{i}$ equal to zero, we obtain

$$
x_{i}=\frac{1}{a_{i}}\left[\sqrt{\frac{a_{i} y_{i}}{\mu_{1}\left(y_{i}+1\right)}}-1\right]^{\dagger}, \quad i=1, \cdots, N_{b}
$$

where $[x]^{\dagger} \triangleq \max (x, 0)$, and $\mu_{1}$ is the solution to the following nonlinear equation

$$
\sum_{i=1}^{N_{b}} \frac{1}{a_{i}}\left[\sqrt{\frac{a_{i} y_{i}}{\mu_{1}\left(y_{i}+1\right)}}-1\right]^{\dagger}=P_{s} .
$$

In a similar fashion, for a fixed $\left\{x_{i}\right\}$ satisfying (23) and (25), we can update $\left\{y_{i}\right\}$ as

$$
y_{i}=\left[\sqrt{\frac{a_{i} x_{i}}{\mu_{2}\left(a_{i} x_{i}+1\right)}}-1\right]^{\dagger}, \quad i=1, \cdots, N_{b}
$$

where $\mu_{2} \geq 0$ is the solutions to the following nonlinear equation

$$
\sum_{i=1}^{N_{b}}\left[\sqrt{\frac{a_{i} x_{i}}{\mu_{2}\left(a_{i} x_{i}+1\right)}}-1\right]^{\dagger}=P_{r} .
$$

Note that the conditional updates of $\left\{x_{i}\right\}$ and $\left\{y_{i}\right\}$ may either decrease or maintain but cannot increase the objective function in (22). Monotonic convergence of $\left\{x_{i}\right\}$ and $\left\{y_{i}\right\}$ follows directly from this observation. After the convergence of the alternating algorithm, $\lambda_{f, i}$ and $\lambda_{b, i}$ can be obtained from (21) as

$$
\lambda_{f, i}=\sqrt{\frac{y_{i}}{\lambda_{s, i}^{2} x_{i}+1}}, \quad \lambda_{b, i}=\sqrt{x_{i}}, \quad i=1, \cdots, N_{b} .
$$

And then the optimal structure of $\overline{\mathbf{F}}$ and $\mathbf{B}$ is given by (13). From (6), we have $\mathbf{H}_{r d, i} \mathbf{F}_{i}=\mathbf{Q} \boldsymbol{\Lambda}_{f} \mathbf{U}_{s, i}^{H}$, where matrix $\mathbf{U}_{s, i}^{H}$ contains the $(i-1) N_{r}+1$ to $i N_{r}$ columns of $\mathbf{U}_{s}^{H}$. Finally $\mathbf{F}_{i}=\left(\mathbf{H}_{r d, i}\right)^{+} \mathbf{Q} \mathbf{\Lambda}_{f} \mathbf{U}_{s, i}^{H}, i=1, \cdots, K$, where $(\cdot)^{+}$denotes matrix pseudo-inverse. 


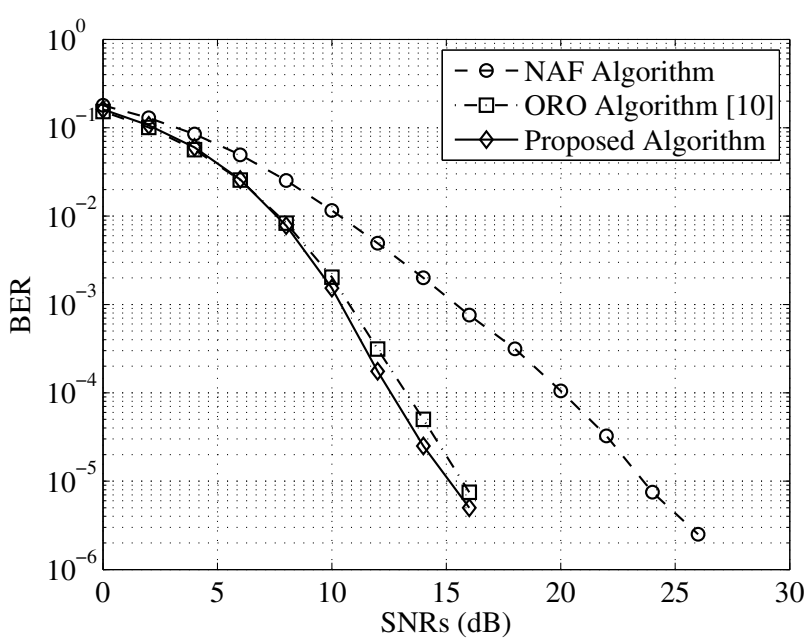

Fig. 3. Example 1. BER versus $\mathrm{SNR}_{\mathrm{s}}$ with $K=3$.

\section{Simulations}

In this section, we study the performance of the proposed optimal joint source and relay beamforming algorithm for parallel MIMO relay systems. All simulations are conducted in a flat Rayleigh fading environment using the BPSK constellation, and the noises are i.i.d. Gaussian with zero mean and unit variance. The channel matrices have zero-mean entries with variances $\sigma_{s}^{2} / N_{s}$ and $\sigma_{r}^{2} /\left(K N_{r}\right)$ for $\mathbf{H}_{s r}$ and $\mathbf{H}_{r d}$, respectively. We vary the signal-to-noise ratio (SNR) in the source-to-relay link $\mathrm{SNR}_{\mathrm{S}}$ while fixing the SNR in the relayto-destination link $\mathrm{SNR}_{\mathrm{r}}$ to $20 \mathrm{~dB}$. We transmit 1000 randomly generated bits in each channel realization, and the bit-error-rate (BER) results are averaged through 200 channel realizations. Here we set $N_{b}=N_{s}=N_{r}=N_{d}=3$.

In the first example, we simulate $K=3$ and compare the BER performance of the proposed joint source and relay algorithm with the naive amplify-and-forward (NAF) algorithm where both the source and relay matrices are scaled identity matrices, and the optimal relay only (ORO) algorithm in [10] which optimizes only the relay amplifying matrices without optimizing the source precoding matrix. From Fig. 3, it can be seen that the NAF algorithm has the worst performance. The proposed algorithm outperforms the other two approaches.

In the second example, we study the effect of the number of relays to the system BER performance using the proposed algorithm. Fig. 4 shows the BER performance with $K=2,3$, and 5. It can be seen that at BER $=10^{-3}$, we achieve a 5-dB gain by increasing from $K=2$ to $K=5$.

\section{CONCLUSions}

In this paper, we have derived the optimal structure of the source precoding matrix and the relay amplifying matrices for parallel MIMO relay communication systems. The proposed source and relay matrices jointly diagonalize the source-relaydestination channel and minimize the MSE of the signal waveform estimation. The proposed algorithm has an improved BER performance compared with existing techniques.

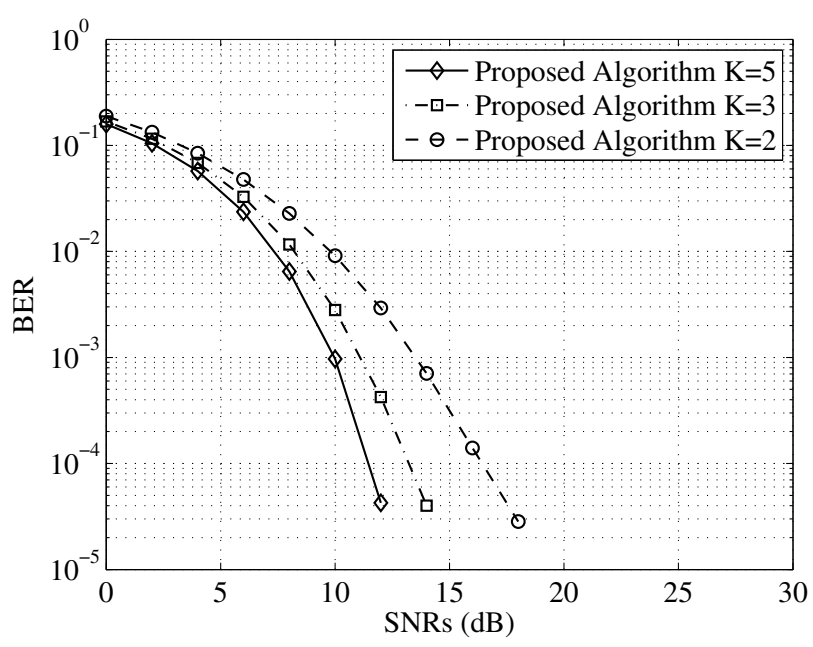

Fig. 4. Example 2. BER versus $\mathrm{SNR}_{\mathrm{S}}$ with varying $K$.

\section{REFERENCES}

[1] H. Bolukbasi, H. Yanikomeroglu, H. Falconer, and S. Periyalwar, "On the capacity of cellular fixed relay networks," in Proc. Canadian Conf. Electrical and Computer Engr., 2004., May 2004, vol. 4, pp. 2217-2220.

[2] R. Pabst, B. H. Walke, D. C. Schultz, D. C. Herhold, H. Yanikomeroglu, S. Mukherjee, H. Viswanathan, M. Lott, W. Zirwas, M. Dohler, H. Aghvami, D. D. Falconer, and G. P. Fettweis, "Relay-based deployment concepts for wireless and mobile broadband radio," IEEE Commun. Mag., vol. 42, pp. 80-89, Sep. 2004.

[3] J. N. Laneman, D. N. C. Tse, and G. W. Wornell, "Cooperative diversity in wireless networks: Efficient protocols and outage behavior," Trans. Inf. Theory, vol. 50, pp. 3062-3080, Dec. 2004.

[4] B. Wang, J. Zhang, and A. Høst-Madsen, "On the capacity of MIMO relay channels," IEEE Trans. Inf. Theory, vol. 51, pp. 29-43, Jan. 2005.

[5] T. Tang, C. B. Chae, R. W. Heath. Jr., "On achievable sum rates of a multiuser MIMO relay channel," in Proc. IEEE ISIT, Seattle, WA, USA, Jul. 2006, pp. 1026-1030.

[6] S. Simoens, O. Muñoz-Medina, J. Vidal, and A. D. Coso, "Compressand-forward cooperative MIMO relaying with full channel state information” IEEE Trans. Signal Process., vol. 58, pp. 781-791, Feb. 2010.

[7] X. Tang and Y. Hua, "Optimal design of non-regenerative MIMO wireless relays," IEEE Trans. Wireless Commun., vol. 6, pp. 1398-1407, Apr. 2007.

[8] O. Muñoz-Medina, J. Vidal, and A. Agustín, "Linear transceiver design in nonregenerative relays with channel state information, "IEEE Trans. Signal Process., vol. 55, pp. 517-519, Jun. 2007.

[9] W. Guan and H. Luo, "Joint MMSE transceiver design in nonregenerative MIMO relay systems," IEEE Commun. Lett., vol. 12, pp. 517-519, Jul. 2008.

[10] A. S. Behbahani, R. Merched, and A. M. Eltawil, "Optimizations of a MIMO relay network," IEEE Trans. Signal Process., vol. 56, pp. $5062-$ 5073, Oct. 2008.

[11] Z. Fang, Y. Hua, and J. C. Koshy, "Joint source and relay optimization for a non-regenerative MIMO relay," in Proc. IEEE Workshop Sensor Array Multi-Channel Signal Process., Waltham, WA, Jul. 2006, pp. 239243.

[12] Y. Rong and Y. Hua, "Optimality of diagonalization of multi-hop MIMO relays," IEEE Trans. Wireless Commun., vol. 8, pp. 6068-6077, Dec. 2009.

[13] Y. Rong, X. Tang, and Y. Hua, "A unified framework for optimizing linear non-regenerative multicarrier MIMO relay communication systems," IEEE Trans. Signal Process., vol. 57, pp. 4837-4851, Dec. 2009.

[14] Y. Rong and F. Gao, "Optimal beamforming for non-regenerative MIMO relays with direct link," IEEE Commun. Letters, vol. 13, pp. 926-928, Dec. 2009.

[15] Y. Rong, "Optimal joint source and relay beamforming for MIMO relays with direct link," IEEE Commun. Letters, vol. 14, pp. 390-392, May 2010. 